\title{
SOBRE LOS DERECHOS MORALES
}

\section{Abismos lingüísticos}

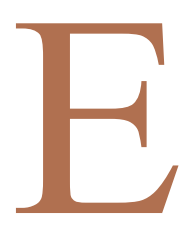

n los últimos años se ha producido en el campo de la filosofía jurídica y moral una cesura cultural curiosa y, en última instancia, deletérea: mientras en la literatura anglosajona ha habido una enorme concentración de atención en los llamados moral rights por autores de muy diversas posiciones, tanto normativas -deontológicos, éticos, conservadores y progresistas, utilitaristas, comunitaristas, marxistas- como metaéticas -realistas, convencionalistas, constructivistas, escépticos-, en la literatura en lengua española se ha formado un fuerte movimiento de resistencia a reconocer el concepto de derechos morales. ${ }^{1}$ Esta cesura es curiosa en tiempos de una creciente universalización del pensamiento teórico en general y filosófico en especial; y es deletérea, ya que traza límites diferentes del discurso que socava las bases para un fructífero intercambio de ideas dentro de un territorio discursivo común.

En algunos casos la resistencia a admitir derechos morales parece puramente lexicográfica: se aduce que en castellano, como en alemán, en italiano y en francés, y a diferencia de lo que ocurre en inglés, se emplea la misma palabra (derecho, Recht, diritto, droit) para referirse al orden jurídico y a los títulos, facultades y permisiones, de modo que la expresión derechos,

${ }^{1}$ Algunos ejemplos están dados por los trabajos de Roberto Vernengo, «Derecho y moral», en Anales de la Cátedra Francisco Suárez, núm. 28/1988, Granada, y «Dos ensayos sobre problemas de fundamentación de los derechos humanos», en Cuadernos de Investigación del Instituto de Investigaciones Jurídicas y Sociales, «Ambrosio L. Gioja», Buenos Aires, 1989; Gregorio Peces-Barba, «Sobre el fundamento de los derechos humanos», en Anales de la cátedra Francisco Suárez, citado, núm. 28/1988, y, con fundamentos diferentes, Eduardo Rabossi, «la fundamentación de los derechos humanos: algunas reflexiones críticas», en $M S$, México, 1987. 
en plural, como sinónimo de facultades, títulos, etcétera está intrínsecamente asociada a la expresión derecho, cuando ella se refiere al orden jurídico, al sistema de normas jurídicas positivas. De este modo, se alega, hablar de derechos jurídicos parece redundante y, por el contrario, referirse a derechos morales parece, si no autocontradictorio, por lo menos paradójico. Esto no se daría en inglés por la distinción entre las palabras right y law para referirse, respectivamente, a las facultades, título y permisiones, por un lado, y al orden jurídico o a las normas jurídicas, por el otro, y, además, por la asociación que allí se da, en cambio, entre el uso de la palabra right con el primer significado y el uso de la misma palabra para referirse a lo correcto o a lo justo.

Esta argumentación lingüística es sumamente desconcertante. No parece prima facie que estas variaciones del léxico puedan tener consecuencias tan profundas como que dos culturas que han permanentemente interactuado tengan una diferencia conceptual tan radical como la que se sugiere: los hablantes del inglés poseerían una categoría que usan ampliamente en su discurso práctico, que nosotros no podríamos reconocer por el accidente de contar con la misma palabra derecho que se usa tanto para denotar un cierto sistema de normas como para hacer referencia a una cierta relación que las normas pueden establecer entre las personas. En segundo término, la explicación de nuestra limitación conceptual no resulta plausible: todos los textos de introducción al derecho explican que el sentido subjetivo y objetivo son dos significados diferentes de la palabra derecho; por lo tanto no se ve porque deberían estar necesariamente asociados si no es por razones fonéticas: hablar de un derecho jurídico no es más redundante que hablar de una prenda que se prenda, ni hablar de un derecho moral es más paradójico que hablar de un cabo de tierra adentro. En tercer lugar, resulta obvio que todo el mundo usa la expresión derecho en contextos no jurídicos («tengo derecho [claramente no jurídico] a que se me escuche», «ahora yo tengo derecho a mover la pieza»), y muchas veces se usa la expresión precisamente para criticar al orden jurídico porque no reconoce el derecho en cuestión (como he sostenido en otro lugar, ${ }^{2}$ ésta es una de las apelaciones más relevantes a los derechos humanos). Finalmente, aun cuando todas las razones anteriores no se aplicasen, si la categoría conceptual que los hablantes del inglés designan con la expresión moral rights

\footnotetext{
${ }^{2}$ Véase Ética y derechos humanos, Buenos Aires, 1989, cap. I.
} 
fuera genuina e importante en el discurso práctico, debería simplemente estipularse una expresión en castellano para aludir a ella, sea derechos morales o cualquier otra (por ejemplo, títulos, exigencias, demandas, facultades, permisos morales), sin hacer de esta cuestión verbal todo un problema filosófico.

De modo que si se cuestiona el reconocimiento de derechos morales, ello no puede ser por limitaciones de nuestro idioma, sino porque se piensa que la categoría conceptual misma es espuria, aun cuando se tenga la suerte de poder denominarla moral rights. Hay que mostrar que para que se dé la relación normativa típica de los derechos las normas que establecen esa relación tienen que tener las características distintivas del derecho.

Pero ¿cuál podría ser el argumento que permita mostrar tal cosa? Dado que la mayoría de los que cuestionan la categoría conceptual de los derechos morales son escépticos en materia ética, alguien podría sostener que lo que se impugna son relaciones normativas establecidas por normas inexistentes. Pero este argumento sería absurdo: el que las normas morales fueran inexistentes haría en todo caso también inexistentes a las relaciones que ellas establecen (y no sólo a los derechos, sino también a los deberes, a las responsabilidades, a las competencias, etcétera), pero esto no quiere decir que no las podamos reconocer conceptualmente así como podemos reconocer las relaciones entre seres mitológicos. Por otra parte, los escépticos y relativistas no suelen negar la existencia de normas morales, sino en todo caso la posibilidad de su fundamentación intersubjetiva y universal, lo que se complementa, generalmente aunque no siempre, ${ }^{3}$ con su identificación con convenciones sociales, o con actitudes subjetivas o con la interpretación de expresiones éticas en términos de expresión de emociones o de formulación de prescripciones. Pero en la medida que, en cualquiera de estas variantes, se le dé alguna interpretación a la existencia de normas morales, ella tendría que extenderse a los derechos que derivan de tales normas.

También se podría sostener que hay alguna otra característica distintiva de las normas jurídicas que hace que ellas y sólo ellas sean generadoras de derechos subjetivos. Por ejemplo, el hecho de que las normas jurídicas, como dice Kelsen, ${ }^{4}$ regulen

${ }^{3}$ Éste no es el caso, por ejemplo, de J. Mackie, quien en Inventing Right and Wrong, Harmondworth, 1977, hace compatible su escepticismo con una teoría ética objetivista.

${ }^{4}$ Véase Teoría Pura del Derecho, trad. Roberto J. Vernengo Nilve, México, 1979, pág. 75. 
sanciones, y, como dicen otros, estén respaldadas por sanciones. Pero la primera caracterización ha sido repetidamente rechazada por no recoger el dato de tantas normas jurídicas que tienen un contenido diverso a la prescripción de sanciones $\mathrm{y}$, lo que me parece más contundente, por haber juicios morales que también califican a actos punitivos como debidos o correctos. Por otra parte, si la supuesta falta de contenido coactivo de las normas morales no es óbice para que ellas generen deberes -que están más directamente asociados con las sanciones, $-{ }^{5}$ no se ve por qué deberían impedir que generen derechos -sobre todo si éstos se ven como correlatos de tales deberes.

Tampoco parece que el que las normas morales no estén respaldadas por sanciones sea óbice para que ellas generen derechos subjetivos. En primer lugar, no es claro que, cuando son aceptadas, no estén respaldadas por sanciones de algún tipo -como el reproche moral- y aun por sanciones ejecutadas a través del aparato coactivo del Estado, sin que por ello se conviertan necesariamente en normas jurídicas. Pero, en segundo término, es menos claro todavía por qué debería exigirse el respaldo de sanciones y aun de sanciones coactivas para que las normas morales generen derechos, cuando ello no ocurre en el caso de los deberes o responsabilidades morales. Sólo si los derechos subjetivos se identificaran con acciones procesales esta posición parecería tener algún sustento, pero, por supuesto, no hay razón para tal identificación, sobre todo cuando ni siquiera los derechos subjetivos jurídicos son completamente reducidos a tales acciones. ${ }^{6}$

Tal vez la vinculación de los derechos subjetivos morales con el respaldo a través de sanciones se justifique como una forma indirecta de decir que para que una relación normativa sea constitutiva de un derecho debe tener efectividad, vigencia, debe gozar de reconocimiento. Cuando ello ocurre la norma que subyace al derecho en cuestión se convierte en una norma social y aun -regla de reconocimiento mediante- en una norma jurídica. Pero cuando ello no ocurre, un derecho que no es efectivo es una mera ficción. Éste no es exactamente el mismo planteo que el anterior relacionado con la existencia de las normas morales, ya que aquí no se trata de su validez intersubjetiva o de la posibilidad de fundamentarlas y adscribirles valores de verdad sino

${ }^{5}$ Véase P. M. S. Hacker, «Sanction Theories of Duty», en Oxford Essays in Jurisprudence, ed. A. W. B. Simpson, Oxford, 1973.

${ }^{6}$ Véase este tema en mi Introducción al análisis del derecho, Buenos Aires, 1980, cap. IV. 
de su vigencia y efectivo reconocimiento. Pero salvo que se identifique la existencia de las normas morales con esa vigencia (con lo que se aceptaría una posición convencionalista) no se ve por qué no se podrían invocar derechos precisamente para obtener su reconocimiento. El discurso habitual en términos de derechos morales -y centralmente el que alude a derechos humanos- no es un discurso constatativo de lo que generalmente se da; es un discurso de reforma y de brega; es un discurso dirigido a adecuar la realidad a ciertos ideales.

Por último, se podría suponer que la resistencia a admitir derechos morales tiene un origen totalmente diverso a los argumentos revisados anteriormente: puede sostenerse que una teoría ética plausible no puede estar basada en derechos, sino en deberes, ideales de lo bueno o en virtudes. Ésta es una vieja controversia que se remonta a Bentham ${ }^{7}$ cuando consideraba a los derechos como «sinsentidos en zancos», pero obviamente aceptaba una teoría ética -el utilitarismo- que está basada en una concepción de lo bueno. El tema fue retomado en trabajos recientes de Mackie, ${ }^{8}$ Dworkin ${ }^{9}$ y Raz, ${ }^{10}$ y la controversia continúa, en la medida en que una teoría moral basada en derechos no es sólo objetada por el utilitarismo, sino también por concepciones aristotélico-tomistas y comunitaristas. ${ }^{41}$ Sin embargo, aunque tal vez haya cierta presuposición no muy consciente en los autores que dudan de los derechos morales de que los deberes o algún ideal del bien -como el utilitarista- son hechos morales más duros que hasta un escéptico podría aceptar como base de una teoría ética, la controversia no está planteada en estos términos de ética normativa, sino como una cuestión conceptual o de metaética. Los escépticos acerca de los derechos morales en el marco de la cultura de habla española se distinguen precisamente de los que rechazan tales derechos en la literatura en idioma inglés por no incursionar en la discusión substantiva de si deberíamos, consecuentemente, aceptar un sistema moral basado en deberes, virtudes o concepciones de lo bueno, generalmente porque también son escépticos acerca de la posibilidad de una discusión racional sobre tales cuestiones.

${ }^{7}$ Véase J. Bentham, The Works of Jeremy Bentham, ed. John Bowring, Edimburgh, 1843, vol. II, pág. 491.

${ }^{8}$ Véase J. Mackie, «Can there be Right-Based Moral Theory», en Theories of Rights, J. Waldron, ed., Oxford, 1984.

${ }^{9}$ Véase R. Dworkin, Taking Rights Seriously, Cambridge, Mass., 1977, cap. 6.

${ }^{10}$ Véase J. Raz, «Right-based Moralities», en Theories of Rights, citada. 
2. El teorema fundamental. -El desarrollo anterior ataca una negativa extrema respecto de los derechos morales que, como hemos visto, resulta bastante poco plausible aun para el escepticismo ético. Pero puede defenderse una tesis más atenuada que se opone a una tesis más fuerte sobre la relevancia de los derechos morales para el razonamiento práctico: la negativa atenuada consiste en sostener que si bien es admisible reconocer conceptualmente a los derechos morales y hasta admitir que las normas que los establecen pueden justificar acciones y decisiones, sin embargo, no puede aceptarse que esas normas que proveen derechos morales tengan, en su campo de aplicabilidad, un carácter justificatorio excluyente. También debemos admitir, según esta posición, que hay proposiciones sobre derechos jurídicos que proveen razones para actuar con total independencia de su posible derivación de principios morales que establecen derechos subjetivos. Se puede agregar que, dado que la mayoría de los derechos nacionales y el derecho internacional reconocen los derechos fundamentales -los derechos humanos- no es necesario, a todos los efectos prácticos que preocupan a quienes se sienten comprometidos por tales derechos, mostrar que tales normas jurídicas derivan de, o están de algún modo apoyadas por, principios morales.

Creo, sin embargo, que tampoco esta negativa atenuada a aceptar derechos morales se justifica, ya que me parece correcta la tesis fuerte opuesta de que los principios morales son excluyentemente justificatorios en su campo de aplicación, y que sólo los derechos establecidos directa o indirectamente por tales principios, y que son por lo tanto derechos morales, pueden servir de razones para actuar.

Esto depende de un tema central, tal vez el tema central de la filosofía del derecho, que es el de determinar en qué medida el discurso jurídico práctico -o sea el discurso que permite justificar acciones y actitudes- es un discurso independiente y autosuficiente o si es parte -o un caso especial, Besonderesfall para usar la expresión de Alexy- ${ }^{11}$ del discurso moral general. En realidad, controversias como la añosa entre iusnaturalismo y positivismo jurídico, que se presentan engañosamente como disputas sobre el concepto de derecho -con lo que se convierten en estériles controversias verbales que desconocen el simple hecho de que puede haber varios de tales conceptos de derecho- ${ }^{12}$ versan sobre esta cuestión

\footnotetext{
${ }^{11}$ Véase R. Alexy, A Theory, of Legal Argumentation, Oxford, 1989.

${ }^{12}$ Véase mi libro La validez del derecho, Buenos Aires, 1985, cap. VII.
} 
Creo que la alternativa del «caso especial» o de la dependencia del discurso jurídico respecto del discurso moral puede ser probada, y lo puede ser de un modo cuasi formal, por lo que me animo a denominar a esta prueba, por sus enormes consecuencias para toda nuestra concepción acerca del derecho, el teorema fundamental de la teoría general del derecho.

Antes de pasar a resumir esa prueba que he desarrollado más extensamente en otro lugar, ${ }^{13}$ debo hacer dos aclaraciones. La primera es que voy a entender por una proposición justificatoria de una acción o decisión a aquella cuya formulación implica cierta inconsistencia práctica con la no realización de la acción o con la adopción de la decisión opuesta. La segunda es que hay diversos conceptos de norma jurídica: puede hacer referencia, por un lado, a una práctica social -o sea a una cierta regularidad de acciones y actitudes-, a un cierto acto lingüístico que se ejecuta con la intención de influir en el comportamiento de otros -como cuando identificamos a las normas jurídicas con prescripciones-, a un cierto texto -como cuando hablamos de interpretar una norma-, y también a las proposiciones que describen a estos fenómenos o entidades, o, por el otro, a un juicio normativo, o sea a una proposición que califica deónticamente a una cierta acción.

Con estas aclaraciones, veamos el caso más claro de un razonamiento jurídico justificatorio que es el que realiza un juez para fundamentar una cierta decisión o una determinada prescripción. Supongamos que la justificación inmediata de, por ejemplo, una orden de lanzamiento, que el juez dicta sea el juicio de que la demandada María debe desalojar la vivienda alquilada. Ella constituye una proposición justificatoria en el sentido de que constituiría una inconsistencia práctica si el juez formulara ese juicio y se negara a dictar la orden de lanzamiento de María o si apoyara la orden de lanzamiento de María en la negación de ese juicio.

En anticipación a la obvia pregunta de cómo el juez fundamenta a su vez el juicio de que María debe desalojar la vivienda alquilada, él dará como razón típicamente la conjunción de dos tipos de juicios: un juicio de hecho del tipo María no pagó dos meses de alquiler y una norma jurídica como el propietario que no recibe dos meses de alquiler tiene derecho a recobrar la vivienda locada. El problema radica en cómo interpretar el juicio que se identifica como una norma jurídica. Como vimos, una

${ }^{13}$ Véase, idem, cap. IX. 
norma jurídica puede concebirse como una práctica social, un acto lingüístico tal como una prescripción o un texto, y también como un juicio que describe la existencia de la práctica social, la prescripción o el texto. Pero en ninguno de estos casos la norma jurídica permitirá por sí sola fundamentar o derivar la proposición justificatoria de la acción o decisión. Se puede describir con verdad que hay una práctica social de desalojar a quien no paga dos meses de alquiler devolviendo al propietario la tenencia del inmueble locado o el acto de alguien de formular una prescripción dirigida a que se desaloje a quien no pague dos meses de alquiler o la existencia de un texto que emplea esas palabras y formular, sin inconsistencia práctica alguna, el juicio justificatorio de que María -que no pagó dos meses de alquiler- no debe ser desalojada. Por supuesto, esto no sería el caso sí la expresión debe en el último juicio hace ella misma referencia sólo a la existencia de una práctica social, prescripción o texto, pero en este caso -en que habría una inconsistencia lógica entre la negación de este juicio y la descripción de la práctica social, prescripción o texto, el juicio María debe ser desalojada no tendría en sí mismo fuerza justificatoria y sería compatible con cualquier acción o decisión.

De modo que para que el juicio el propietario que no cobra dos meses de alquiler tiene derecho a recuperar la tenencia de su inmueble locado permita justificar la proposición justificatoria María debe ser desalojada él sólo puede ser una norma jurídica en el cuarto sentido que vimos al comienzo: el sentido que alude a un juicio normativo, o sea una proposición que califica a una conducta como prohibida, permitida u obligatoria. Por cierto que este juicio normativo que permite justificar directa o indirectamente una acción o decisión debe distinguirse de una prescripción: ésta consiste en el acto de formular un juicio normativo con la intención de que esa formulación -dado quien la hace o las condiciones en que se la hace- sirva como razón auxiliar para actuar a cierta persona, dadas sus otras razones operativas. ${ }^{14}$

Una vez que aceptamos que el fundamento de una proposición justificatoria particular en un típico razonamiento práctico jurídico sólo puede ser una norma jurídica entendida como un juicio normativo, es razonable plantearse la pregunta acerca de cómo sabemos que ese juicio normativo en el que el juez se basa

${ }^{14}$ Véase este concepto en J. Raz, Practical Reasons and Norms, Londres, 1975, cap, I. 
para fundamentar su decisión es, al fin y al cabo, una norma jurídica. Porque el juicio el propietario que no recibe dos meses de alquiler tiene derecho a recuperar el inmueble locado bien puede ser interpretado como un juicio moral, por ejemplo cuando es formulado por alguien que ignora lo que dispone el orden jurídico, o le resulta indiferente lo que él disponga, o sabe que el orden jurídico no dispone nada al respecto -tiene una laguna-, o sabe que dispone lo opuesto y sugiere modificarlo. Kelsen diría que un juicio normativo como el del ejemplo será identificado como una norma jurídica o como un juicio moral tomando en cuenta si lo que él determina como debido o permitido es o no un acto coactivo, o una sanción. En este caso, si el desalojo es visto en tales términos, el juicio en cuestión podría ser considerado una norma jurídica. Pero ya vimos en el punto anterior que este criterio distintivo de las normas jurídicas no es plausible, no sólo porque hay obvias normas jurídicas que no disponen sanciones sino, sobre todo, porque hay claros juicios morales que califican como permitidos o debidos a actos coactivos que tienen el carácter de penas (como, por ejemplo, «los narcotraficantes deben ser sometidos a pena de muerte»). En el ejemplo anterior también vimos obvios contextos en que el juicio el propietario que no cobre dos meses de alquiler tiene derecho a recuperar la tenencia de su inmueble locado puede ser interpretado como un juicio moral.

Si el contenido no es distintivo de los juicios normativos jurídicos deberá serlo entonces su origen. Y, en efecto, parece claro que lo que distingue a los juicios normativos jurídicos de los morales es el hecho de ser aceptados, en el razonamiento práctico que justifica una acción o decisión, por el hecho de haber sido formulados por cierta autoridad, que puede ser legislativa o convencional. De modo que un juicio del tipo el propietario que no cobra de su inquilino dos meses de alquiler tiene derecho a recuperar su inmueble locado sólo es concebido como una norma jurídica cuando es aceptado en el razonamiento práctico, de un juez, por ejemplo, por la razón de haber aceptado previamente un juicio del tipo el legislador L ha prescripto que el propietario que no cobra dos meses de alquiler tiene derecho a recuperar la tenencia de su inmueble. Sólo si se acepta el primer juicio porque se acepta este segundo tipo de juicio el primero es concebido como una norma jurídica.

Pero ya hemos visto que el segundo tipo de juicio consiste en la descripción de una norma jurídica entendida como una prescripción (también podría ser la descripción de la práctica social consistente en aceptar la prescripción) y que ese tipo de juicio no 
tiene fuerza justificatoria: él es pragmáticamente compatible con cualquier acción o decisión. Por lo tanto, no es lógicamente posible que alguien acepte el primer tipo de juicio por la razón de aceptar el segundo tipo de juicio; si lo hiciera incurriría en un obvio non sequitur. A lo sumo, un juicio del tipo el legislador L ha prescripto... sólo puede ser parte de la razón de que se acepte un juicio del tipo el propietario tiene derecha a... La parte operativa de la razón tiene que estar dada por otro juicio con fuerza justificatoria del tipo el legislador $L$ debe ser obedecido o tiene autoridad o derecho para emitir prescripciones.

Llegados a este punto, cabe la obvia pregunta acerca de si este último juicio normativo, que constituye el núcleo de la razón operativa por la cual una norma jurídica es aceptada, es en sí mismo una norma jurídica. Y aquí la respuesta tendrá que recorrer de nuevo todo el camino que ya hemos visto: no puede ser distinguido como norma jurídica por su contenido sino por la razón por la cual es aceptado; esa razón no sólo debe incluir la descripción de lo prescripto por una autoridad o convención sino un juicio normativo que les da autoridad o legitimidad. Por supuesto que el proceso no puede proseguir al infinito y llegará un punto en que debemos aceptar que una norma jurídica es aceptada en virtud de un juicio normativo que no es una norma jurídica, puesto que no es a su vez aceptado por haber sido formulado por una autoridad que otro juicio normativo legitima sino por sus propios méritos. Ahora bien, un juicio normativo que es aceptado no por haber sido formulado por cierta autoridad sino por sus propios méritos, es precisamente lo que se suele identificar como un juicio moral -en esto consiste precisamente el rasgo de autonomía de los juicios morales enfatizado por Kant.

Con esto tenemos la conclusión aparentemente paradójica de que las normas jurídicas se identifican como tales por su derivación en el razonamiento práctico de juicios de índole moral. Si un juicio que deriva lógicamente de un juicio moral también lo es, de aquí se sigue que las normas jurídicas, entendidas como proposiciones justificatorias, son un caso especial de juicios morales. Por cierto, que Kelsen ${ }^{15}$ advirtió perfectamente este riesgo de confusión entre el derecho y la moral y pretendió evitarlo mediante el recurso de sostener que el juicio normativo último que no es aceptado por derivar de cierta fuente dotada de autoridad

\footnotetext{
${ }^{15}$ Véase op. cit., cap. V.
} 
-su famosa norma básica- no es un juicio moral puesto que su fuerza justificatoria queda neutralizada por el hecho de que no es aceptado categóricamente sino hipotéticamente en el razonamiento jurídico. Pero un juicio normativo que es aceptado sólo como hipótesis sólo permite justificar decisiones hipotéticas, por lo que no tiene ningún rol en un razonamiento práctico justificatorio de acciones y decisiones reales, como el de los jueces.

Creo que esto constituye una demostración de que las normas jurídicas que establecen derechos -por ejemplo el juicio de el propietario tiene derecho a recobrar el inmueble locado a quien no ha pagado dos meses de alquiler- sólo tienen un carácter justificatorio de acciones o decisiones si constituyen una especie de juicios morales puesto que derivan de un juicio moral que legitiman a cierta autoridad y de un juicio que describe la prescripción de esa autoridad. Si convenimos en que los derechos que están establecidos por normas morales son derechos morales, de aquí se sigue que sólo los derechos morales permiten justificar acciones o decisiones.

De este modo, intenté mostrar que ni siquiera es correcta la tesis débil que niega que los derechos morales sean los únicos derechos a los que puede aludirse para criticar o defender una acción o decisión. Esto es, por supuesto, consecuencia, de la tesis más amplia de que el discurso jurídico justificatorio es «un caso especial» o es dependiente del discurso moral. Esta tesis más amplia tiene la consecuencia -desagradable, por cierto, para los escépticos en materia ética- de que la presunta subjetividad, relatividad o imposibilidad de fundamentación de los presupuestos del discurso moral se transmite al discurso jurídico justificatorio.

3. Relaciones entre moral y derecho.- Quienes se oponen a la idea de que sólo derechos de índole moral tienen carácter justificatorio de acciones y decisiones señalan también la obscuridad que presenta la alegada relación entre normas morales y normas jurídicas, lo que se manifiesta en la incertidumbre acerca de qué relaciones tiene que haber entre derechos establecidos por las primeras y los establecidos por las últimas: por ejemplo, un comportamiento, como el de circular por la derecha puede ser moralmente indiferente (¿permitido?), pero jurídicamente obligatorio; puede haber comportamientos que están moralmente prohibidos o que son obligatorios (mentir, ayudar a un amigo necesitado) pero que son jurídicamente facultativos y son, por lo tanto objeto de derechos.

Las relaciones lógicas entre normas morales y jurídicas y entre 
los derechos establecidos por ellas son por cierto intrincadas y deberían ser objeto de más atención de los especialistas de lógica deóntica, una vez que superen los prejuicios en contra de los principios y derechos morales. Sin embargo, hay algunas cosas que surgen con cierta obviedad hasta para un lego en esa disciplina.

En primer lugar, la demostración anterior indica que la relación entre normas jurídicas -entendidas como juicios normativos o como razones justificatorias- y las normas morales es una relación de identidad de caso, es decir, hay casos de normas morales que son normas jurídicas, puesto que la clase de las normas jurídicas justificatorias es una especie de la clase de normas morales; no todo principio o juicio moral es una norma jurídica, pero toda norma jurídica que tiene un rol de razón operativa en el razonamiento práctico es un juicio moral especial, que se distingue por derivar de un principio moral que legitima a cierta fuente de prescripciones y de una descripción de una de tales prescripciones. Por lo tanto, no se presentan los problemas que algunos autores advierten en las relaciones de derivación o subalternación entre normas jurídicas y normas morales; tampoco puede objetarse la duplicación del contenido de ambos tipos de normas, precisamente porque son las mismas.

Claro está que uno puede distinguir entre normas morales puras, en cuya aceptación no es en absoluto relevante la prescripción de una autoridad, y normas jurídico-morales, que son aquellas que son aceptadas en virtud de que una norma moral pura legitima la prescripción de cierta autoridad. Como vimos, para que el segundo tipo de normas establezca derechos que permiten justificar acciones o decisiones ellas deben derivar de normas morales puras. Pero esto no quiere decir que esas normas morales puras también deben establecer derechos: ellas pueden establecer simplemente el deber de obedecer a cierta autoridad o pueden valorar positivamente las consecuencias de obedecer a tal autoridad. No tiene por qué haber, entonces, una relación de derivación entre los derechos morales que surgen como consecuencia de la prescripción de una autoridad y derechos que surgirían de normas morales puras que legitiman tal autoridad (un utilitarista negaría este último tipo de derechos morales como elementos irreducibles del sistema moral, pero no tendría por qué negar el primer tipo de derechos morales). Cuando una autoridad legitimada por normas morales puras prescribe el deber de realizar un cierto comportamiento, los beneficiarios de ese comportamiento tienen un derecho -según algunas posiciones ello sólo es así cuando, además, se tiene una 
acción procesal para hacerlo cumplir. ${ }^{16}$ Estos derechos correlativos de deberes que están establecidos por normas jurídico-morales son los que Dworkin llama «derechos institucionales». ${ }^{17}$

Pero además, por supuesto, las normas morales puras también pueden establecer directamente derechos, que los podemos llamar derechos fundamentales para distinguirlos de los derechos institucionales, que también son de índole moral. Es un tema sumamente controvertido si esos derechos son irreducibles o si deben ser interpretados, por ejemplo, también como correlativos de deberes o como derivados de ciertos ideales del bien. Tengo para mí que estos derechos no institucionales no pueden ser interpretados en el contexto de una concepción liberal de la sociedad como reflejos de deberes de otros sino como fundamento de tales deberes, una vez que se dan ciertas condiciones adicionales como es la posibilidad de actuar; creo que lo que esos derechos exigen es que cierto estado de cosas valioso deba ser obtenido, lo que es diferente que decir que hay un deber de producirlo (como se advierte en inglés con la diferencia entre las palabras ought y duty). ${ }^{18}$ También es una cuestión controvertible de ética sustantiva si los derechos fundamentales determinan sólo deberes de los legisladores o directamente también de los jueces y de los ciudadanos en general.

Por cierto que, dependiendo de qué posición se tome en controversias como las mencionadas, puede haber conflictos entre las normas morales puras y las normas jurídico-morales, y entre los derechos fundamentales y los institucionales (y los deberes) que ellas establecen. Estos conflictos reflejan tensiones entre las normas morales puras de tipo substantivo y la norma moral pura que otorga legitimidad a una cierta autoridad o práctica social. Es una cuestión de ética normativa determinar cómo se resuelven estos conflictos, de modo que prevalezcan uno u otro tipo de normas puras. Por ejemplo, intuitivamente estamos dispuestos a sostener que prevalece el derecho institucional establecido por una autoridad legítima de no desalojar un inmueble debido a una suspensión general de los desalojos, por una situación de emergencia económica, sobre lo que algunos conciben como un derecho fundamental de propiedad que excluye cualquier uso ajeno no consentido. Sin embargo, hay otros casos en que sostendremos que prevalece el derecho fundamental

\footnotetext{
${ }^{16}$ Véase mi libro Introducción al análisis del derecho, citado, cap. IV.

${ }^{17}$ Véase, op. cit., cap. 4.

${ }^{18}$ Véase mi libro citado, Ética y derechos humanos, cap. I.
} 
establecido por normas morales puras sobre el derecho moral-institucional (por ejemplo, si el primero protege bienes como la vida o la integridad corporal). En otro lugar ${ }^{19}$ he tratado de desarrollar una teoría general que pretende ofrecer criterios para resolver este tipo de conflictos.

Es obvio que también podemos hablar de derechos en contextos no justificatorios y referidos a normas jurídicas concebidas como prácticas sociales, prescripciones o textos. En estos casos las proposiciones que hacen referencia a derechos serán meramente descriptivas y no proporcionaran razones para justificar una acción o decisión (así podemos decir, por ejemplo, que nadie tenía derecho a más de una comida diaria en los campos de concentración nazis). Por supuesto, estas proposiciones son completamente independientes de juicios morales normativos. Pero pueden interactuar con ellos en el marco del razonamiento práctico. Hemos visto precisamente que los juicios que permiten derivar normas jurídicas justificatorias -o sea juicios jurídico-morales- de principios morales puros que legitiman a cierta autoridad, son de este tipo, puesto que son juicios que describen la prescripción de cierta autoridad o determinada práctica social. Debe ser sumamente complejo, y no parece que pueda resolverse sin ciertos criterios de ética sustantiva, determinar cuándo un derecho en este sentido puramente descriptivo da lugar a un derecho moral institucional dado cierto trasfondo de normas morales puras -las que a su vez, como vimos, pueden establecer derechos fundamentales, o sea, no institucionales.

Por otro lado, las normas jurídicas entendidas como prácticas sociales, prescripciones o textos o las proposiciones descriptivas que dan cuenta de ellas tienen otra función crucial en el razonamiento práctico, además de la de permitir la derivación de normas jurídico-morales a partir de normas morales puras: cuando aquellas normas han tenido un origen democrático creo, como lo he tratado de mostrar en otro lugar, ${ }^{20}$ que constituyen un fundamento epistémico para inferir con alguna probabilidad -mayor o menor de acuerdo a la amplitud y pureza del procedimiento democrático pero nunca con certeza absoluta- cuáles son las normas morales puras. Esto introduce una enorme complicación en la determinación de la relación entre derechos fundamentales, derechos en sentido puramente descriptivo y derechos institucionales, ya que los derechos puramente descriptivos

\footnotetext{
${ }^{19}$ Véase, idem.

${ }^{20}$ Véase, ibídem, cap. IX.
} 
pueden tanto constituir una justificación epistémica para inferir derechos morales fundamentales como permitir, además, la derivación lógica a partir de ellos de derechos morales-institucionales.

Esto puede llegar a constituir un intrincado rompecabezas lógico, pero me parece que ello no es motivo para excusar la resistencia a admitir derechos morales. 